EPJ Web of Conferences 111, 07002 (2016)

DOI: $10.1051 /$ epjconf/201611107002

(C) Owned by the authors, published by EDP Sciences, 2016

\title{
Fission Rate Ratios of FCA-IX Assemblies as Integral Experiment for Assessment of TRU's Fission Cross Sections
}

\author{
Masahiro Fukushima ${ }^{1, a}$, Kazufumi Tsujimoto ${ }^{1}$, and Shigeaki Okajima ${ }^{1}$ \\ ${ }^{1}$ Japan Atomic Energy Agency (JAEA), 2-4 Shirakata, Tokai-mura, Naka-gun, Ibaraki, Japan, 319-1195
}

\begin{abstract}
At the fast critical assembly (FCA) of JAEA, central fission rate ratios for TRU such as ${ }^{237} \mathrm{~Np},{ }^{238} \mathrm{Pu},{ }^{239} \mathrm{Pu},{ }^{242} \mathrm{Pu},{ }^{241} \mathrm{Am},{ }^{243} \mathrm{Am}$, and ${ }^{244} \mathrm{Cm}$ were measured in the seven uraniumfueled assemblies (FCA-IX assemblies) with systematically changed neutron spectra. The FCA-IX assemblies were constructed with simplicity both in geometry and composition. By virtue of these FCA-IX assemblies where the simple combinations of uranium fuel and diluent (graphite and stainless steel) in their core regions were systematically varied, the neutron spectra of them cover from the intermediate to fast one. Taking their advantages, benchmark models with respect to the central fission rate ratios had been recently developed for the evaluation of the TRU's fission cross sections. As an application of these benchmark models, the Japanese Evaluated Nuclear Data Library JENDL-4.0 was utilized by a Monte Carlo calculation code. Several results show large discrepancies between the calculation and experimental values. The benchmark models would be well suited for the evaluation and modification of the nuclear data for the TRU's fission cross sections.
\end{abstract}

\section{Introduction}

In studies on the evaluation and modification of nuclear data, integral and differential experiments have complementarity relations. The measured data in integral experiments apparently lack information of neutron energy since they are integrated out by weighting of neutron spectra. However, the set of integral data measured in a variety of neutron spectra has a potential to be useful for the evaluation of cross sections since the sensitivities of the measured data show energy dependence.

Seven uranium-fueled assemblies (FCA-IX assemblies) with systematically changed neutron spectra were constructed at the FCA in the 1980s, and the central fission rate ratios (CFRRs) were measured for seven TRU nuclides containing minor actinides, ${ }^{237} \mathrm{~Np},{ }^{238} \mathrm{Pu},{ }^{239} \mathrm{Pu},{ }^{242} \mathrm{Pu},{ }^{241} \mathrm{Am},{ }^{243} \mathrm{Am}$, and ${ }^{244} \mathrm{Cm}$ [1-2]. Each assembly is identified by a number (1-7) after the "IX," e.g., IX-1 for the first one. Each was a coupled system comprising a central core region and a surrounding blanket region with depleted uranium metal blocks. They were simple, both in geometry and composition. Each core region was composed of a simple combination of high enriched uranium (HEU) and graphite in the assemblies IX-1 to IX-3 and that of HEU and stainless steel in the assemblies IX-4 to IX-6. Their neutron spectra cover from the intermediate to fast ones by varying the combinations of fuel and loaded diluent. In addition, the assembly IX-7 was constructed as a low enriched uranium (LEU)

\footnotetext{
${ }^{\text {a }}$ Corresponding author: fukushima.masahiro@jaea.go.jp
} 
assembly without diluent. The three-dimensional benchmark models of the FCA-IX assemblies were recently developed with respect to the criticalities [3] and the CFRRs [4]. The purpose of this paper is to introduce the analysis results of CFRRs of the FCA-IX assemblies with using the new benchmark models, from a viewpoint of integral experiment for the nuclear data evaluation. In Section 2, we briefly review the benchmark models with respect to the CFRRs. In Section 3, we discuss the Monte Carlo calculation results by JENDL-4.0 [5]. Finally, the conclusion is shown in Section 4.

\section{Brief review of CFRRs of FCA-IX assemblies}

\subsection{Experiments}

The fission rates for seven nuclides, ${ }^{237} \mathrm{~Np}$ (F37), ${ }^{238} \mathrm{Pu}$ (F48), ${ }^{239} \mathrm{Pu}(\mathrm{F} 49),{ }^{242} \mathrm{Pu}(\mathrm{F} 42),{ }^{241} \mathrm{Am}$ (F51), ${ }^{243} \mathrm{Am}$ (F53), and ${ }^{244} \mathrm{Cm}$ (F64), were measured at the core center of each FCA-IX assembly [1-2]. Seven fission chambers of parallel-plate type [6] were used. Figures 1 and 2 illustrate the structure of the fission chamber and the loading of fission chamber into the central core drawer, respectively. The deposit mass was determined within $\pm 1.5 \%$ error $\left( \pm 3.0 \%\right.$ for $\left.{ }^{244} \mathrm{Cm}\right)$ by $\alpha$-spectrometry using silicon surface barrier detector mounted in a vacuum chamber. The uncertainties in the CFRRs measurements are due to those in the $\alpha$-assay of the deposit mass, the statistical counting errors, errors introduced by the impurity isotope corrections. As a typical example, Table 1 shows uncertainties of F37, F49, and F64 in the assembly IX-1. The uncertainties in the $\alpha$-assay mainly contribute to the total ones.

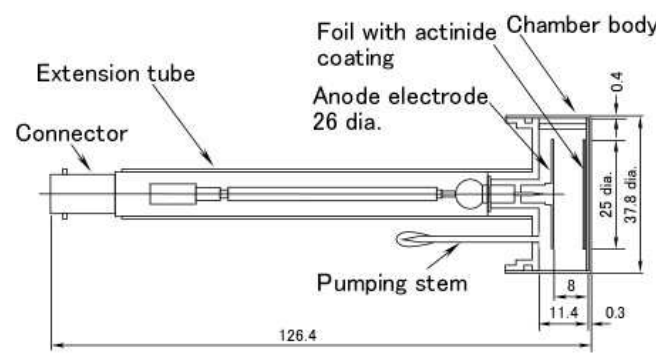

Dimensions in $\mathrm{mm}$

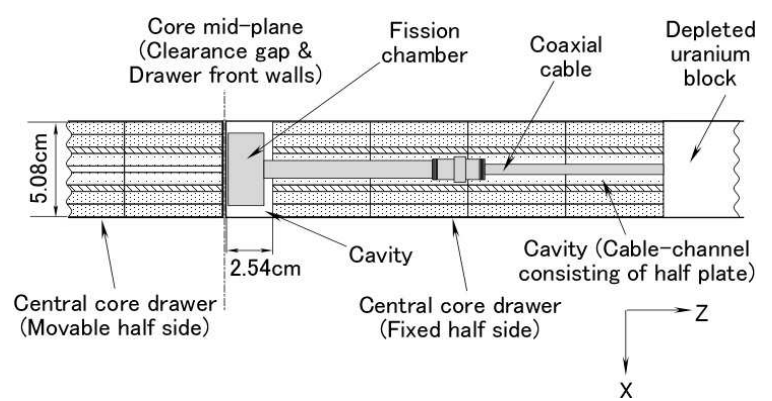

Figure 2. Loading of fission chamber.

Figure 1. Structure of fission chamber.

Table 1. Components of uncertainties of typical fission rates in the assembly IX-1.

\begin{tabular}{|l|c|c|c|}
\hline & $\mathrm{F} 37$ & $\mathrm{~F} 49$ & $\mathrm{~F} 64$ \\
\hline$\alpha$-assay of the deposit mass & $1.5 \%$ & $1.5 \%$ & $3.0 \%$ \\
\hline Statistical counting error & $0.4 \%$ & $0.4 \%$ & $0.8 \%$ \\
\hline Impurity isotope correction & - & $0.3 \%$ & $1.0 \%$ \\
\hline Total uncertainty & $1.6 \%$ & $1.6 \%$ & $3.3 \%$ \\
\hline
\end{tabular}

\subsection{Benchmark models and Calculations}

Even though the FCA-IX assemblies are simple in geometry and composition, we should be taken account for the geometric conditions during the measurement in analysis of the CFRRs. To develop the benchmark models as simple as possible, we took care not to increase the total uncertainty far beyond the original experimental one. In reference [4], the new three-dimensional benchmark models for the CFRR analysis were developed by simplifying geometric details while keeping the plate-wise heterogeneity due to the plate arrangement in the core drawers. The CFRRs calculated in this new benchmark models, $\mathrm{C}_{\text {bench }}$, were compared with the values $\left(\mathrm{E}_{\mathrm{bench}}\right)$ corresponding to the measured ones $\left(\mathrm{E}_{\text {exp }}\right)$ with taking account for the following two simplification effects: (a) the first one is relating to geometric modelling of the CFRR measurement conditions, i.e. the fission chamber and the cavities 
occupied in the central core drawer and so on, and (b) the second one is relating to that of other complex conditions specific to the FCA facility as mentioned in the reference [3]. Each simplification effect was estimated by the Monte-Carlo code with continuous neutron energy, MVP [7], as described below.
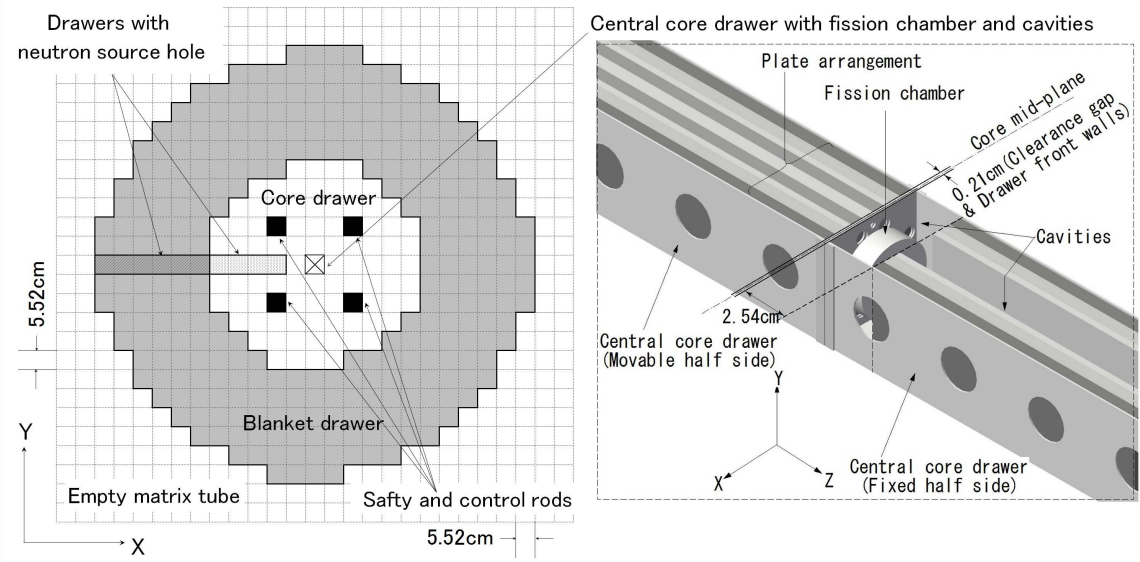

Figure 3. Configurations of as-built model for the assembly IX-1.
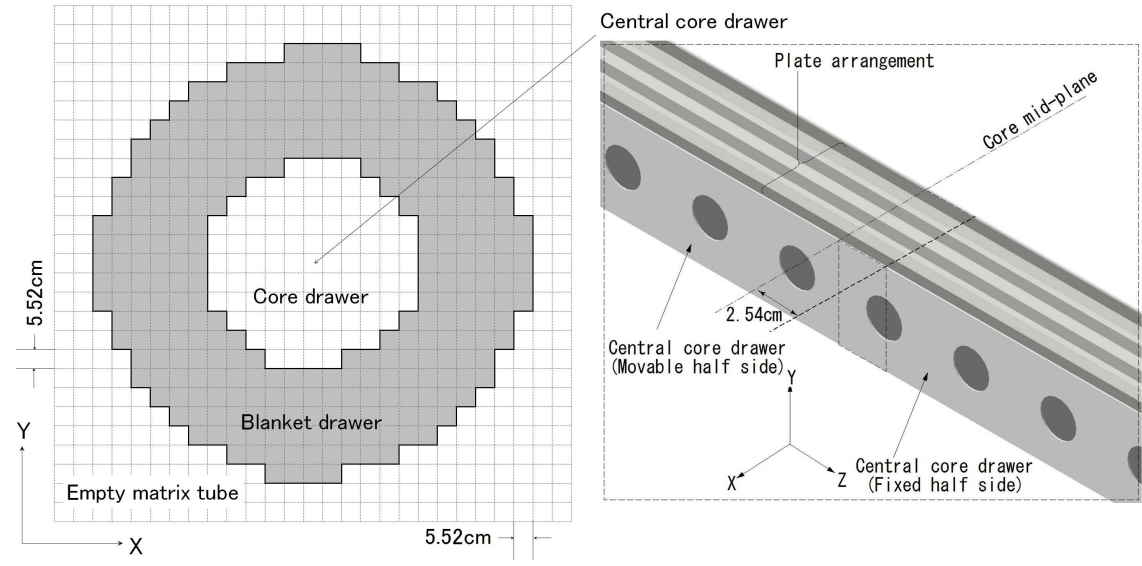

Figure 4. Configurations of benchmark model for the assembly IX-1.

\section{(1) Step 1}

As the as-built calculations, the experimental configuration with the fission chamber and the cavities and so on was modelled as detailed as possible by MVP. Figure 3 illustrates the XY-configuration and the bird's eye view of the central core drawer of the as-built model for the assembly IX-1. To calculate the CFRR, the cylindrical space inside the fission chamber was adopted as the tally. The calculated CFRR with this model is denoted as $C_{\text {as-built }}$.

(2) Step 2

As for the CFRR measurement conditions, the central drawer with the fission chamber and the cavities was simplified to be same as the core drawers in the core region. To calculate the CFRR, the amounts of fissionable nuclide corresponding to the fission chamber were doped into the matrix tube and drawer at the core center ranging from the core mid-plane to $2.54 \mathrm{~cm}$ along the Z-direction. This model could be used in deterministic calculations. The calculated CFRR in this model is denotes as $C_{\text {simple }(a) \text {. }}$

\section{(3) Step 3}

The experimental configuration includes other complex conditions such as the clearance gap and the drawer front walls at the core mid-plane, the structure of the safety control rods, and the hole for 
neutron source insertion as also shown in Figure 3. As the second simplification, these geometries were simplified while keeping the plate-wise heterogeneity due to the plate arrangement in the core drawers. Figure 4 illustrates the XY-configuration and the bird's eye view of the central core drawer of the benchmark model for the assembly IX-1. The calculated CFRR in this simple model is denoted as $C_{\text {simple }(b)}$.

These simplification effects were indicated as the ratios, $f_{a}=C_{\text {simple(a) }} / C_{\text {as-built }}$ and $f_{b}=C_{\text {simple(b) }} / C_{\text {simple(a) }}$. For each ratio calculation by MVP, the history number was 200 million. The benchmark CFRR value (E $\mathrm{E}_{\text {bench }}$ ) was finally obtained by multiplying these effects, $f_{a}$ and $f_{b}$, to the measured one ( $\mathrm{E}_{\text {exp }}$ ). As a typical example of the results, Table 2 summarizes the measured CFRRs $\left(\mathrm{E}_{\text {exp }}\right)$, the simplification effects, and the benchmark CFRRs $\left(\mathrm{E}_{\text {bench }}\right)$ in the assembly IX-1. The results for the other assemblies IX-2 to IX-7 are given in reference [4]. The simplification effects are within a few percent in total and the increase of uncertainty of the CFRR is negligible small for all the assemblies.

The further information related to the homogenization and its effect were provided in reference [4] together with the homogenous atomic number densities and the three-dimensional configuration of each FCA-IX assembly.

Table 2. Measured CFRRs, simplification effects, and benchmark CFRRs of the assembly IX-1.

\begin{tabular}{|c|c|c|c|c|}
\hline \multirow{2}{*}{ Type } & \multirow{2}{*}{$\begin{array}{c}\text { Measured CFRR } \\
\left(E_{\text {exp }}\right)\end{array}$} & \multicolumn{2}{|c|}{ Simplification effects } & \multirow{2}{*}{$\begin{array}{c}\text { Benchmark CFRR } \\
\left(\mathrm{E}_{\text {bench }}\right)\end{array}$} \\
\hline & & $f_{a}=C_{\text {simple }(a)} / C_{\text {as-built }}{ }^{*}$ & $f_{b}=C_{\text {simple }(b)} / C_{\text {simple }(a)}{ }^{* 2}$ & \\
\hline F37/F49 & $0.2048 \pm 2.2 \%$ & $0.9842 \pm 0.0046$ & $1.0031 \pm 0.0032$ & $0.2022 \pm 2.3 \%^{* 3}$ \\
\hline F48/F49 & $0.5856 \pm 2.4 \%$ & $0.9948 \pm 0.0050$ & $1.0008 \pm 0.0034$ & $0.5830 \pm 2.5 \%$ \\
\hline F42/F49 & $0.1584 \pm 2.2 \%$ & $0.9834 \pm 0.0046$ & $1.0031 \pm 0.0032$ & $0.1562 \pm 2.3 \%$ \\
\hline F51/F49 & $0.1961 \pm 2.2 \%$ & $0.9831 \pm 0.0047$ & $1.0037 \pm 0.0033$ & $0.1935 \pm 2.3 \%$ \\
\hline F53/F49 & $0.1509 \pm 2.2 \%$ & $0.9822 \pm 0.0047$ & $1.0038 \pm 0.0033$ & $0.1488 \pm 2.3 \%$ \\
\hline F64/F49 & $0.2614 \pm 3.6 \%$ & $0.9866 \pm 0.0045$ & $1.0022 \pm 0.0031$ & $0.2585 \pm 3.7 \%$ \\
\hline
\end{tabular}

${ }^{*}$ Simplification effect relating to modelling of the CFRR measurement conditions. ${ }^{* 2}$ Simplification effect relating to modelling of the other complex geometries. ${ }^{* 3}$ Estimated by error propagation of the measurement uncertainties and the Monte Carlo statistical errors in $C_{a s-b u i l t}$ and $C_{\text {simple(b) }}$.

\section{Discussions of benchmark results}

The benchmark calculations by JENDL-4.0 were carried out and the results are shown in Table 3. The calculations for F37/F49 agreed with the experimental values $\left(\mathrm{E}_{\mathrm{bench}}\right)$ for all the assemblies. On the other hand, the calculations for F64/F49 obviously overestimate by $11 \%-18 \%$ and the $\mathrm{C}_{\text {bench }} / \mathrm{E}_{\text {bench }}$ values slightly increase with neutron spectrum hardening from the assemblies IX-1 to IX-4. For the other CFRRs, the results are summarized below:

- For F48/F49, the calculations underestimate about by $5 \%$ for all the assemblies,

- For F42/F49, the calculations overestimate about by $8 \%$ for all the assemblies,

- For F51/F49 and F53/F49, the calculations underestimate about by $4 \%$ and $6 \%$, respectively.

Table 3. Benchmark calculation results $\left(\mathrm{C}_{\text {bench }} / \mathrm{E}_{\text {bench }}\right)$ with MVP and JENDL-4.0 [4].

\begin{tabular}{|c|c|c|c|c|c|c|c|}
\hline Type & IX-1 & IX-2 & IX-3 & IX-4 & IX-5 & IX-6 & IX-7 \\
\hline F37/F49 & $0.994 \pm 0.023^{*}$ & $0.996 \pm 0.027$ & $0.991 \pm 0.025$ & $1.004 \pm 0.025$ & $0.994 \pm 0.025$ & $0.992 \pm 0.020$ & $0.999 \pm 0.025$ \\
\hline F48/F49 & $0.953 \pm 0.024$ & Not measured & $0.961 \pm 0.026$ & $0.956 \pm 0.026$ & $0.955 \pm 0.026$ & $0.954 \pm 0.026$ & $0.955 \pm 0.026$ \\
\hline F42/F49 & $1.077 \pm 0.025$ & $1.071 \pm 0.029$ & $1.081 \pm 0.027$ & $1.086 \pm 0.022$ & $1.090 \pm 0.022$ & $1.081 \pm 0.022$ & $1.090 \pm 0.027$ \\
\hline F51/F49 & $0.968 \pm 0.022$ & $0.947 \pm 0.027$ & $0.963 \pm 0.024$ & $0.961 \pm 0.024$ & $0.957 \pm 0.024$ & $0.962 \pm 0.024$ & $0.958 \pm 0.024$ \\
\hline F53/F49 & $0.939 \pm 0.022$ & $0.933 \pm 0.025$ & $0.945 \pm 0.024$ & $0.934 \pm 0.023$ & $0.938 \pm 0.023$ & $0.943 \pm 0.024$ & $0.948 \pm 0.024$ \\
\hline F64/F49 & $1.112 \pm 0.041$ & $1.135 \pm 0.044$ & $1.152 \pm 0.043$ & $1.165 \pm 0.043$ & $1.168 \pm 0.043$ & $1.175 \pm 0.046$ & $1.159 \pm 0.044$ \\
\hline
\end{tabular}

As a typical case in the results, we discuss the large overestimations of F64/F49 as following. The previous work [2] shows that the C/E values of F64/F49 by JENDL-3.3 range from 1.01 to 1.05. From these results, the calculation values of F64/F49 become about $10 \%$ larger by changing from JENDL3.3 to JENDL-4.0. Figure 5 shows the sensitivity coefficient of F64/F49 to ${ }^{244} \mathrm{Cm}$ fission cross 
section. The sensitivity coefficient is mainly distributed above the fission threshold energies around 1 $\mathrm{MeV}$ for all the assemblies. Figure 6 compares the ${ }^{244} \mathrm{Cm}$ fission cross sections between JENDL-4.0 and JENDL-3.3. The ${ }^{244} \mathrm{Cm}$ fission cross section of JENDL-4.0 around $1 \mathrm{MeV}$ is about $10 \%$ larger than that of JENDL-3.3, which results in the large overestimations of F64/F49 by JENDL-4.0.

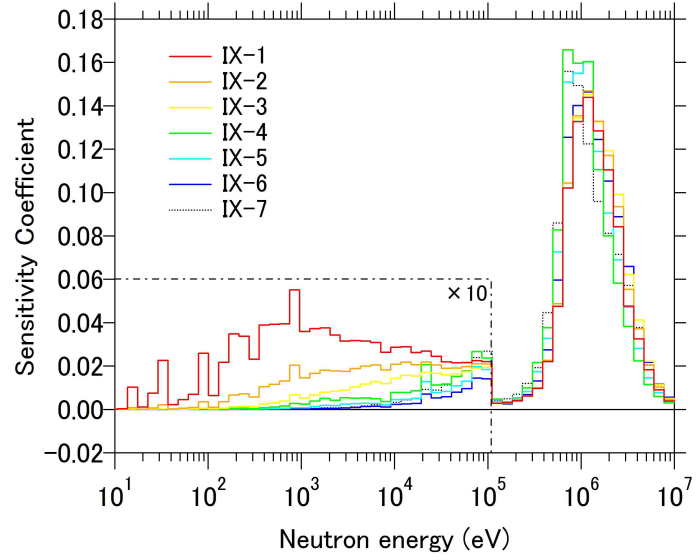

Figure 5. Sensitivity of F64/F49 to ${ }^{244} \mathrm{Cm}$-fission.

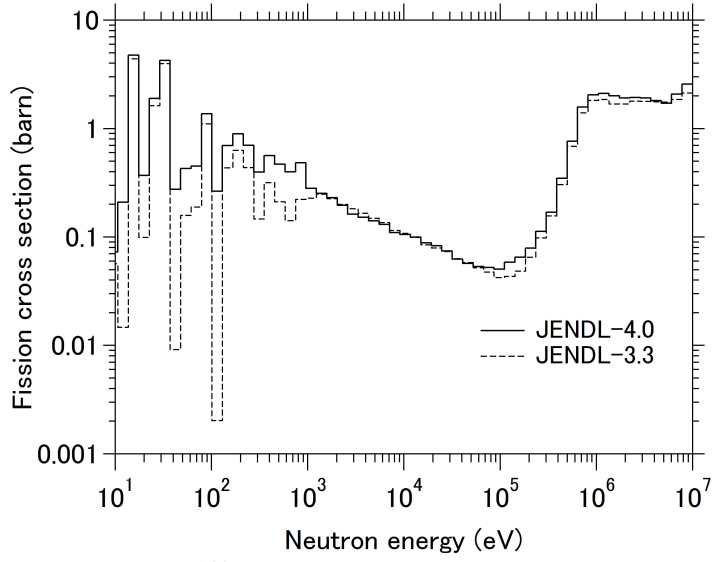

Figure 6. ${ }^{244} \mathrm{Cm}$-fission cross sections.

\section{Conclusion}

In this paper, we reviewed the three-dimensional benchmark models for the CFRRs of the FCA-IX assemblies with systematically changed neutron spectra and discussed benchmark results by JENDL4.0. The new benchmark models were developed without increasing the total uncertainty far beyond that of the original experiment. The benchmark results for F37/F49 show that the calculations by JENDL-4.0 agreed with the experimental values for all the assemblies. For F64/F49, the large overestimations by JENDL-4.0 are found for all the assemblies, which is caused by the large fission cross section of ${ }^{244} \mathrm{Cm}$ above the fission threshold energies around $1 \mathrm{MeV}$. For the other CFRRs, F48/F49, F42/F49, F51/F49, and F53/F49, the results by JENDL-4.0 show discrepancies between the calculation and experimental values. The benchmark models would be well suited for the evaluation and modification of the nuclear data for these fission cross sections owing to their advantages of simplicity and systematically changed neutron spectra.

\section{Acknowledgements}

We would like to thank Mr. T. Mukaiyama for valuable information about the CFRR measurements of the FCA-IX assemblies.

\section{References}

1. T. Mukaiyama, M. Obu, M. Nakano, et al., Proc. of Int. Conf. on Nuclear Data for Basic and Applied Science, Santa Fe, USA, 483 (1985)

2. S. Okajima, M. Fukushima, T. Mukaiyama, Proc. of International Conference on the Physics of Reactors (PHYSOR2008), Interlaken, Switzerland (2008)

3. M. Fukushima, Y. Kitamura, T. Kugo, et al., J. Nucl. Sci. Technol., Published online, (2015)

4. M. Fukushima, A. Oizumi, H. Iwamoto, et al., JAEA-Data/Code 2014-030 (2015) [in Japanese]

5. K. Shibata, O. Iwamoto, T. Nakagawa, et al., J. Nucl. Sci. Technol., 48, 1 (2011)

6. M. Obu, JAERI-M 9757 (1981) [in Japanese]

7. Y. Nagaya, K. Okumura, T. Mori, et al., JAERI 1348 (2005) 
